\title{
DIE BRUSTFLOSSE DES GRÖNLANDSWALES (BALAENA MYSTICETUS L.)
}

\author{
VON \\ W. KÜKENTHAL, \\ BERLIN. \\ (Mit einer Tafel.)
}

Das Studium der Brustflosse des Grönlandswales hat zu Fragen von allgemeinerem Interesse geführt. Ihre kurze und breite Form sowie ihre anscheinende Fünffingerigkeit, die ausserdem nur noch beim südlichen Glattwal (Balaena australis) vorkommt, liess sie ursprünglicher erscheinen als die schlankere und durchweg vierfingerige Brustflosse aller übrigen Bartenwale, bei denen also ein Finger verloren gegangen sein muss. Auch die Entscheidung darüber, welcher Finger verschwunden ist, war leicht gefällt, ist doch beim Grönlandswal der „Daumen” bereits ein rudimentäres Gebilde. So kam man zu dem Schlusse, dass es der erste Finger ist, der allen anderen Bartenwalen fehlt. Es war daher keine geringe Ueberraschung, als ich 1890 an den Brustflossen eines Embryo vom Finwal (Balaenoptera physalus L.) zwischen den beiden mittleren Fingern, gleichweit von jedem entfernt, einen Knorpelstab entdeckte, der aus drei bis vier Phalangen bestand, von der gleichen leicht eingeschnürten Sanduhrform, wie sie für die Bartenwale charakteristisch ist. Die Deutung dieses Gebildes als rudimentärer Finger war unabweisbar, und der Umstand, dass das Interstitium, in dessen distalem Teile er lag, von zwei Aesten des Nervus medianus versorgt wurde, die benachbarten nur von einem, schien mir ein vollgültiger Beweis dafür, dass es nicht der Daumen, sondern der Mittelfinger ist, der bei den Bartenwalen verloren gegangen ist. Der rudimentäre Mittelfinger ist bis jetzt in 9 Fällen und zwar sowohl beim erwachsenen Finwal wie auch bei Embryonen verschiedenster Grösse aufgefunden worden, stets gleichweit von den beiden benachbarten Fingern entfernt, und niemals in irgend welcher Verbindung mit einem von beiden. Von einer sekundären Abspaltung, wie sie bei manchen Zahnwalen in Erscheinung tritt, kann also keine Rede sein. Letztere von M. BRAUN (1907) und dann von seinem Schüler A. KUNZE (1912) vertretene Auffassung habe ich vor kurzer Zeit eingehend zurückgewiesen (1921) und gezeigt, dass die bei Zahnwalen entstandenen Neubildungen mit dem Fingerrudiment beim Finwal nicht das geringste $z u$ tun haben. In seiner verdienstlichen Arbeit über die Brustflosse der Bartenwale erhebt KunZE selbst den Einwand, dass es doch erstaunlich wäre, wenn bei den Furchenwalen einerseits der Daumen ausfalle, andrerseits aber zwischen den mittleren Fingern wiederum eine fingerartige Neubildung auftrete; er sucht diesen sehr berechtigten Einwand mit dem Hinweise zu entkräften, dass die landbewohnenden Vorfahren der Bartenwale, schon ehe sie sich dem Wasserleben anpassten, vierfingerig waren, oder aber dass die Brustflossen einen Funktionswechsel vom Lokomotions- zum Steuerorgan durchzumachen hatten, dem ein Wechsel in der Entwicklungsrichtung parallel ging. Als weitere Stütze seiner Auffassung, dass es doch der Daumen wäre, der bei den Bartenwalen verloren gegangen sei, führt er die Anordnung der Muskulatur ins Feld, sowie den Umstand, dass beim Seihwal auch das dritte Interstitium eine doppelte Innervation vom N. ulnaris erhalte. Eine entgültige Entscheidung dieser interessanten Frage können wir erwarten, wenn wir das Handskelett des Grönlandswales in seiner Entwicklung kennen lernen, um zu entscheiden, ob dessen sogenannter Daumen wirklich den 
ersten Finger darstellt. Bis jetzt ist von diesem Handskelett folgendes bekannt: In ihrer grundlegenden Monographie über den Grönlandswal geben EsCHRICHT und REINHARDT (1861) eine gute Abbildung des Handskelettes vom Erwachsenen, an der sich der Daumen als kurzes, dem Radiale aufsitzendes Knorpelstück mit einem kleinen Knochenkern erkennen lässt. Das Gebilde ist etwas nach innen eingekrümmt und wird als Metacarpus des Daumens bezeichnet. An einem Neugeborenen und zwei grossen Foeten konnten die Verfasser aber keine Spur einer Trennung zwischen diesem Gebilde und dem darunter liegenden Knorpelelement wahrnehmen, und kommen zu dem Schlusse, dass es unsicher ist, ob man überhaupt von einem Daumen beim Grönlandswal reden kann, solange dessen Carpus noch gänzlich knorpelig ist. An zwei grösseren foetalen Flossen, die ich (1893 p. 250) abbildete und beschrieb, fand ich an der einen ebenfalls eine fast völlige Verschmelzung des Daumens mit dem darunter liegenden Radiale vor; zum Unterschied vom Erwachsenen sass das Gebilde aber mehr seitlich dem Radiale an, während der benachbarte Finger mit seinem proximalen Metacarpalende in voller Breite mit der oberen horizontalen Fläche des Radiale artikulierte. Da ein solches radial dem Carpus ansitzendes Knorpelstück auch bei den Furchenwalen vorkommt, wo es als Präpollex bezeichnet wird, nahm ich keinen Anstand, auch beim Grönlandswal den vermeintlichen Daumen mit dem Präpollex zu identifizieren. Neuerdings meint WINGE (1919, p. 119), dass dieser merkwürdigen Deutung wohl mein Wunsch zu Grunde gelegen hätte, eine Uebereinstimmung mit Balaenoptera zu erzielen, bei der ich des Glaubens wäre, nachgewiesen zu haben, dass nicht der Daumen, sondern der dritte Finger verloren gegangen sei. Ohne Beibringung eigener Beobachtungen erklärt er das von mir aufgefundene Rudiment des dritten Fingers für ein paar losgerissene Fingerglieder, von denen es nicht zweifelhaft sein könne, dass es sich um eine Missbildung handle. Wenn ich es nunmehr unternehme, meine Deutung auf embryologischem Wege zu begründen, so weiss ich sehr wohl, dass ich darauf verzichten muss, auch WINGE davon zu überzeugen.

Wäre der sogenannte „Daumen” beim Grönlandswal wirklich ein rudimentär werdender Finger, so müsste er unbedingt in seinen Entwicklungsstadien vollkommener ausgebildet sein, und seine Fingernatur deutlicher zeigen, als beim Erwachsenen. Dagegen sprechen schon die von ESCHRICHT und REINHARDT und später von mir gemachten Angaben, nunmehr bin ich aber in der Lage, an einem relativ jungen Entwickelungsstadium den bündigen Nachweis zu führen, dass dieses Gebilde überhaupt kein Finger ist.

Ein in meinem Besitz befindlicher Embryo vom Grönlandswal von nur $52 \mathrm{~cm}$. Rückenlänge, dessen Körperform ich bereits 1914 beschrieben und abgebildet habe, zeigt eine etwas andere Form der Brustflossen als jene, die wir beim Erwachsenen kennen. Bei letzterem verhält sich die grösste Breite zur Länge wie $1: 2$, bei unserem Embryo aber nur wie 3:5. Die Brustflosse muss also in späterer embryonaler Zeit bedeutend mehr in die Breite als in die Länge wachsen, oder mit anderen Worten, die grosse Breite der Balaenaflosse ist eine sekundäre Erscheinung. Diese Feststellung ist deshalb von Wert, weil dadurch die von WINGE vertretene Auffassung, dass die grosse Breite der Balaenaflosse ein ursprüngliches Merkmal sei, als unrichtig nachgewiesen wird, und weil es daher nicht mehr angeht, dieses Merkmal als Beweis dafür zu benutzen, dass die Glattwale, gegenüber den Furchenwalen mit ihren schmaleren Brustflossen, die ursprünglicheren seien.

Die beiden embryonalen Flossen wurden nunmehr auf ihr Handskelett hin präpariert, die rechte von der Dorsalseite, die linke von der Ventralseite aus. Beiden Flossen fehlt der angebliche „Daumen” überhaupt! Zunächst glaubte ich wenigstens an der rechten Flosse noch ein sehr kleines fingerähnliches Gebilde (siehe Fig. 1) als den sogenannten Daumen ansprechen zu müssen, es erwies sich aber bei weiterer Präparation als eine ausschliesslich aus Bindegewebe bestehende, schlauchartige Vorstülpung ohne eine Spur von Knorpel darin; an der linken Flosse war auch von diesem bindegewebigen Schlauche nichts mehr zu sehen. Es wies also an beiden Extremitäten der Knorpel keinen fingerartigen Vorsprung am radialen Carpusrande auf, und wir sehen nur dem Carpus radial angefügt ein kleines Knorpelelement, eben den Praepollex, der als Grundlage für ein sekundäres Auswachsen zum sogenann„Daumen” des Grönlandswales dient.

Fragen wir uns nun auf welche Weise sich ein solches sekundäres Auswachsen zu einem fingerähnlichen Gebilde erklären lässt, so müssen wir uns an die erst in späterer Embryonalzeit, längst nachdem sich die 4 echten Fingeranlagen knorpelig und z. T. verknöchert ausgebildet haben, 
erfolgende Verbreiterung der Flosse erinnern, die sich besonders auf der Höhe des Carpus geltend macht; infolge dieser rücken beide Flossenränder von dem sie stützenden Handskelett ab, und diẹ Ausbildung von ein paar neuen seitlichen Flossenstützen wird notwendig, um die Flossenränder gespannt und damit die Brustflosse voll funktionsfähig zu erhalten. Die Grundlagen zur Ausbildung dieser neuen Stützen sind aber gegeben ulnar im Pisiforme, das sich bei Balaena stark vergrössert, und radial durch den Praepollex, der ebenfalls seitlich auswächst und dadurch fingerähnlich wird. Eine gewisse Biegsamkeit, welche zur Funktion als Flossenstütze erforderlich ișt, wird durch eine lang dauernde Verknorpelung und geringe Verknöcherung gewährleistet, und eine Zerlegung in einzelne Glieder ist nicht notwendig. Das sehen wir bei Balaena mysticetus. Es ist aber sehr wohl denkbar, dass bei zunehmender Grösse der Flossenstütze der gleiche Prozess des Zerfalls in einzelne Glieder eintritt, wie bei den echten Fingern, da dadurch die Biegsamkeit der Stütze erhöht wird; dahẹr ist es sehr wohl möglich, dass bei Balaena australis dieser Zustand eingetreten ist und die radiale Flossenstütze fingerartig gegliedert ist. Doch muss ich darauf hinweisen, dass die bisherigen Angaben, soweit sie auf Untersuchungen an präparierten und montierten Skeletten beruhen, durchaus nicht zuverlässig sind. An frischem Material vorgenommene Untersuchungen am Nordkaper, Balaena glacialis, der nach neueren Forschungen artlich identisch ist mit Balaena australis, haben ergeben (siehe R. C. ANDREws), dass der sogenannte Daumen stets ungegliedert war.

Wenn mir vom Nordkaper auch kein embryologisches Material zur Verfügung steht, so ist bei dessen naher Verwandtschaft mit dem Grönlandswal mit Sicherheit zu erwarten, dass auch bei ihm der Daumen nicht dem ersten Finger entspricht, sondern eine sekundäre Neubildung wie beim Grönlandswal darstellt.

Somit ist festgestellt, dass die Hand der Glattwale ebenso wie die der Furchenwale vierfingerig ist, und es ist zu erwarten, dass auch bei ersteren der Mittelfinger verloren gegangen ist.

Noch ein paar Worte über den Carpus unseres Embryos. Die Carpalia. sind ähnlich angeordnet wie beim Erwachsenen. Das Radiale ist deutlich und scharf abgegrenzt gegen den Präpollex, im Gegensatz zu den Befunden an grösseren Foeten, wo Verschmelzungen stattgefunden haben. Das grosse Ulnare dürfte auch die beiden distalen Carpalia 4 und 5 enthalten. In der distalen Reihe kommt nur ein Element vor, das unter dem Metacarpale des zweiten Fingers liegt und daher wohl als $\mathrm{C}_{2}$ bezeichnet werden kann. Die Carpalia sind noch ohne Knochenkerne, während diese in den Metacarpalien bereits vorhanden sind, wie die beiden Abbildungen zeigen. Uebrigens sind die Deutungen der einzelnen Knorpelstücke nur provisorische. Die Entwickelung des Handskelettes von Megaptera, über die ich kürzlich berichtet habe, hat mir gezeigt, wie irrig die Deutungen sein können, wenn man nicht die ersten Anlagen beobachten kann.

Nunmehr zur Muskulatur der Hand übergehend, die sich trotz des sehr mässigen Erhaltungszustandes des seit vielen Jahren in Alkohol aufbewahrten Materiales noch leidlich gut präparieren liess, beginne ich mit der Dorsalseite, die an der rechten Extremität freigelegt wurde (siehe Fig. 2). Zwei dicht nebeneinander laufende Muskeln zeigen sich an der. Innenseite von Elle und Speiche, von denen der Ulnare der breitere ist und aus zwei Portionen besteht, die sich etwa in der Mitte des Unterarmes vereinigen. Die aus ihm entspringende starke Sehne teilt sich auf der Höhe des distalen Ulnaendes in drei Aeste, die zu den drei ulnaren Fingern ziehen. Am schwächsten entwickelt ist der des zweiten Fingers, der sich mit zwei' bis drei vom radialen Muskel kommenden Aesten zu einer gemeinsamen Fingersehne vereinigt. Ausserdem versorgt die vom radialen Muskel kommende Sehne den ersten Finger. Der zweite Finger allein ist es also, dessen Sehne sowohl vom ulnaren wie vom radialen Muskel her Wurzeln empfängt. Alle Fingersehnen laufen bis zum Fingerende und strahlen besonders an den angeschwollenen Phalangenenden in fächerartig angeordnete, zur Insertion dienende Fasern aus.

Ganz ähnlich schildert StRUTHERs (1878) die Anordnung bei einem jüngeren Exemplare des Grönlandwales; bei einem voll erwachsenen liegen die Verhältnisse etwas anders, indem bei diesem alle vier Finger von einer gemeinsamen, sich in 4 Aeste spaltenden Sehne überzogen werden. Das gleiche ist nach Kunze bei den von ihm untersuchten Furchenwalen der Fall. Wir können daher nur annehmen, dass die beiden ursprünglich getrennten Streckermuskeln sich später zu einem von KunZE als $M$. extensor digitorum communis bezeichneten gemeinsamen Muskel vereinigen. Da der Zustand beim Embryo des Grönlandswales zweifellos der ursprünglichere ist, darf daher nur der ulnare Muskel, 
dessen Sehnen den zweiten bis fünften Finger versorgen, entsprechend dem Verhalten bei den Landsäugetieren, als gemeinsamer Fingerstrecker bezeichnet werden, während der erste Finger seinen eigenen Fingerstrecker hat, der einen Anteil seiner Sehne zum zweiten herüberschickt, selbst aber keinen Anteil vom zweiten Finger her erhält.

Wenn Kunze schreibt, dass, falls bei den Bartenwalen der dritte Finger wirklich ausgefallen wäre, die Sehnen des Extensor digitorum communis wahrscheinlich nur zu den drei ulnaren, aber nicht $\mathrm{zu}$ dem radialen Finger treten würden, so liefert das Verhalten beim embryonalen wie beim jugendlichen Grönlandswal den schönsten Beweis, dass das in der Tat der Fall ist, und eine treffliche Stütze für die Annahme des Ausfalls des dritten Fingers.

Andere auf dem Präparat und dessen photographischer Wiedergabe auftretende Muskeln sind der $M$. flexor carpi ulnaris, der vom Olecranon zum Pisiforme zieht, sowie der schwächere M. abductor digiti quinti, der vom Pisiforme zum Metacarpale des fünften Fingers reicht. An den angeblichen Daumen tritt weder beim Erwachsenen noch beim Embryo eine Sehne heran!

Wir kommen nunmehr zu der Beugerseite der Hand, die an der linken Flosse freigelegt wurde (siehe Fig. 3). Am Unterarm verlaufen zwei vollkommen voneinarder getrennte Muskeln, ein radialer schwächerer, der den ersten Finger versorgt und einen schwachen Ast zur Sehne des zweiten Fingers entsendet, und ein kräftigerer, ulnarer Muskel, der auf der Höhe der distalen Carpalia drei Aeste für die drei ulnaren Finger abgibt. Der radiale, den ersten Finger versorgende Muskel kann nur der M. flexor pollicis longus sein, der ulnare, die drei ulnaren Finger, versorgende, ist der $M$. flexor digitorum ulnaris. Beim erwachsenen Grönlandswal findet nach STRUTHERS ein Austausch von Sehnenfasern beider Muskeln statt, indem von der Sehne des zweiten Fingers ein Ast zum ersten Finger. zieht, der etwa zu 9/10 vom radialen, zu 1/10 vom ulnaren Muskel versorgt wird. Darin müssen wir, ebenso wie auf der Streckerseite ein sekundäres Verhalten sehen. Ganz ähnlich liegen nach KüNzes Beschreibung und Abbildung (Taf. 83, fig. 3) die. Verhältnisse beim Seihwal, wo ebenfalls ein gegenseitiger Austausch von Sehnenfasern des ersten und des zweiten Fingers vorhanden ist. Beide Muskeln werden von ihm als Einheit zusammengefasst, und als $M$. flexor digitorum profundus bezeichnet, der nach meinen Ausführungen aus dem Flexor digitorum ulnaris und dem Flexor pollicis longus zusammengesetzt ist.

Das Präparat klärt auch über die Innervation der Hand auf, wobei zu berücksichtigen ist, dass auf der photographischen Abbildung die Nerven sich weniger scharf von den sie beleitenden Blutgefässen unterscheiden, wie am Präparat, wo sie schon' durch ihre glänzend weisse Farbe auffallen. Zwischen Radius und Ulna liegt ein starkes Bündel von Nerven, der Nervus medianus, von dem ein Ast das erste Interstitium versorgt, während das zweite Interstitium zwei nahe der Innenkante der Finger verlaufende Aeste aufweist, und das dritte. wiederum nur einen Ast enthält, der in dessen Mitte verläuft und in verschiedener Höhe schwache Seitenzweige abgibt. Der Verlauf des Nervus ulnaris liess sich bei dem mässigen Erhaltungszustande des Objektes nur in seinem proximalen Teile verfolgen, am Unterarm an der ulnaren Kante der Ulna gelegen, dann unter den Ansatz des Pisiforme ziehend. Nur ein Ast an der ulnaren Seite des fünften Fingers korinte festgestellt werden, nicht aber die Verteilung im dritten Interstitium. Jedenfalls ersehen wir aber, dass wie bei den anderen daraufhin untersuchten Bartenwalen so auch beim Grönlandswal der Nervus medianus zwei Aeste in das zweite Interstitium entsendet. Da dieses Verhalten einer doppelten Innervation bei anderen Säugetieren nicht vorkommt, ein paar noch nicht sicher gestellte Ausnahmen abgerechnet, so darf hierin wohl ein Beweis erblickt werden, dass in diesem Interstitium ein Finger verloren gegangen ist. Der von KunZE festgestellte Fall, dass beim Seihwal das Interstitium zwischen viertem und fünftem Finger eine doppelte Innervation vom N. ulnaris (in meiner Abhandlung über die Brustflosse des Buckelwales (1921) steht irrtümlich $N$. medianus) hat, schwächt die Beweiskraft durchaus nicht, handelt es sich ja doch um einen anderen Nerven, und kann diese doppelte Innervation durch den $N$. ulnaris auch auf einer anderen Ursache wie dem Schwunde eines Fingers beruhen.

Fassen wir die Resultate unserer Untersuchung kurz zusammen, so ergibt sich folgendes:

1) Das beim erwachsenen Grönlandswal vorhandene fingerartige Gebilde an der radialen Seite des Handskeletts ist nicht der erste Finger, sondern eine Neubildung, die jüngeren Entwicklungsstadien noch völlig fehlt und erst allmählich durch Auswachsen eines als Präpollex zu bezeichnenden radialen Carpalelementes entsteht. 
2) Die fingerartige Ausbildung dieses Präpollex wird hervorgerufen, durch eine sekundäre Verbreiterung der Brustflosse, deren verbreiterte Ränder einer Unterstützung durch seitliche Skeletteile bedürfen, die durch Verlängerung von Praepollex und Pisiforme geschaffen werden. Die Breite der Brustflosse des Grönlandswales darf also nicht als ein. ursprüngliches Merkmal angesehen werden.

3) Die Hand des Grönlandswales ist vierfingerig, wie die der anderen Bartenwale auch, und damit ist ein durchgreifender Unterschied gegenüber der fünffingerigen Zahnwalhand gegeben.

4) Beim Finwal hatte ich nachgewiesen, dass es der Mittelfinger ist, der verloren gegangen ist. Das bei diesem aufgefundene Fingerrudiment ist keinesfalls durch Abspaltung oder als Missbildung entstanden.

5) Wenn auch beim Grönlandswal kein solches Rudiment eines dritten Fingers mehr auftritt, so beweist doch die Anordnung der Muskulatur, wie die Innervation der Interstitien, dass nur dieser verloren gegangen sein kann.

6) Bei allen Bartenwalen sind demnach die vier vorhandenen Finger mit dem ersten, 'zweiten, vierten und fünften Finger der übrigen Säugetiere zu homologisieren.

\section{ZITIERTE LITERATUR}

1861 Eschricht und ReinhardT. Om Nordhvalen (Balaena mysticetus L.) in: Vidensk. Selsk. Skr. S. raekke v. 5.

1878 Struthers, J. Account of rudimentary fingermuscles found in the Greenland Right-Whale. Journ. Anat. Physiol. v. 12. 1890 KüKenthal, W. Cetologische Notiz. Anat. Anz. 1890, p. 709.

1893 - - Vergleichend-anatomische und entwicklungsgeschichtliche Untersuchungen an Waltieren 2. Bd., Jena. Denkschr. 1895 Struthers, J. On the carpus of the Greenland Right Whale, etc. in: Journ. Anat. Phys. v. 29.

1907 BRAUN, M. Ueber das Brustflossenskelett der Cetaceen in: Schr. Phys. oekon. Ges. in Königsberg, 48. Jahrg.

1912 Kunze, A. Die Brustflossen der Wale. Zool. Jahrb. Anat. v. 32.

1914 KüKENTHAL, W. Untersuchungen an Walen. Zweiter Teil. Jena. Denkschr.

1919 WingE, H: Udsigt over Hvalernes indbyrdes Slaegtskab in: Vidensk. Medd. Dansk. Naturh. Forening v. 70.

1921 KüKenthal, W. Die Brustflosse des Buckelwales (Megaptera nodosa Bonnat.) und ihre Entwicklung, Sitz. Ber. Ak. Wiss. Berlin.

1922 _- Zur Stammesgeschichte der Wale. Sitz. Ber. Akad. Berlin. 


\section{ERKLÄRUNG DER ABBILDUNGEN}

Fig. 1. Rechte Brustflosse des Embryos von Balaena mysticetus, von der dorsalen Seite her praepariert. Vergr. $3: 2$.

Fig. 2. Handskelett und Muskulatur der Streckerseite der rechten Brustflosse. Vergr. $3: 1$.

Fig. 3. Handskelett, Muskulatur und Innervation der. Beugerseite der linken Brustflosse. Vergr. $3: 1$. 


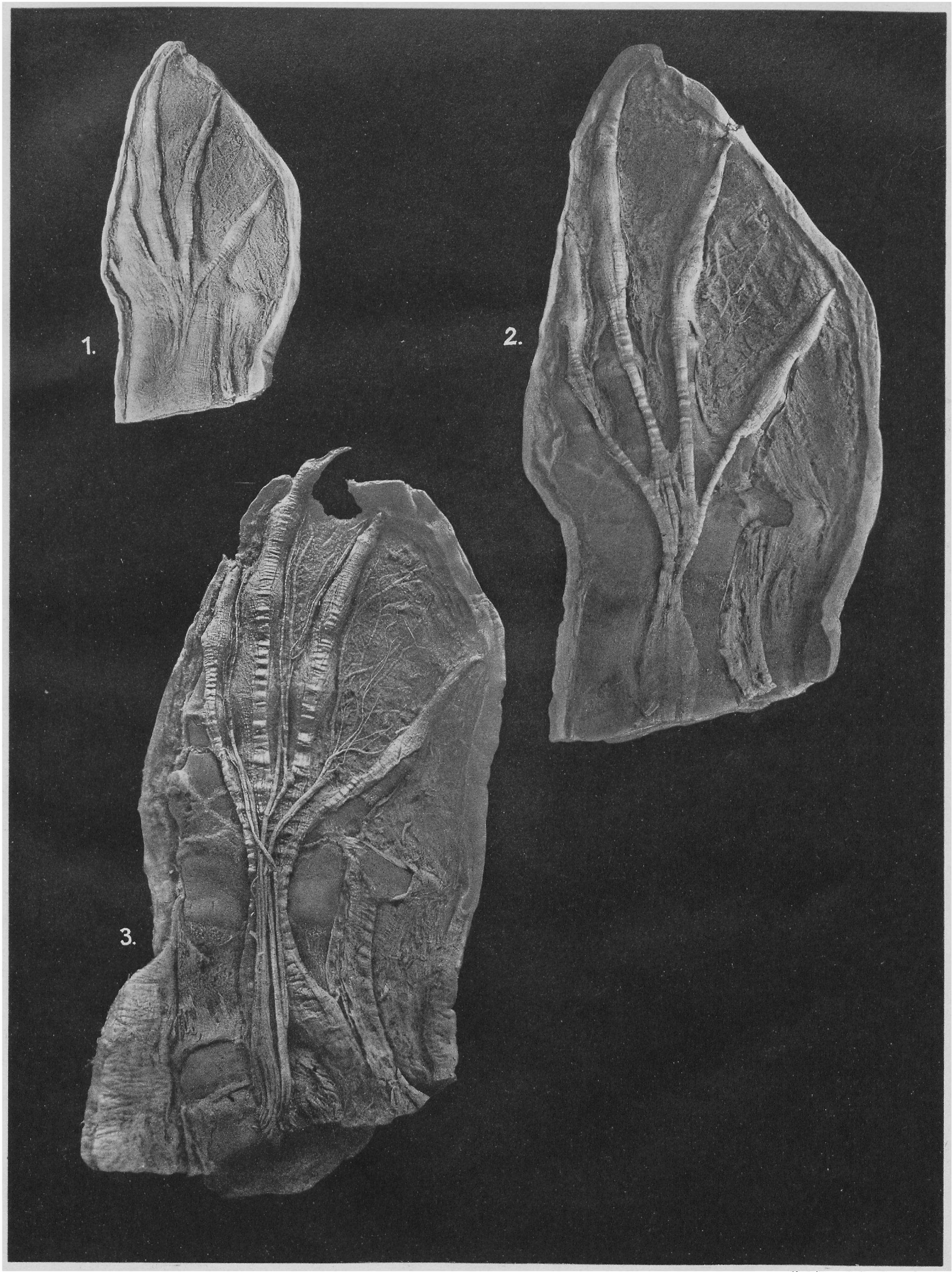

